\title{
Computed Tomography on Renal Masses in Dogs and Cats
}

Kazuaki YAMAZOE, Fumihito OHASHI ${ }^{1)}$, Tsuyoshi $\mathrm{KADOSAWA}^{2)}$, Ryohei NISHIMURA ${ }^{2)}$, Nobuo SASAKI ${ }^{2)}$, Akira TAKEUCHI ${ }^{3)}$

Department of Veterinary Surgery, Faculty of Agriculture, Gifu University, 1-1 Yanagido, Gifu 501-11, ${ }^{1)}$ Department of Veterinary Surgery, College of Agriculture, University of Osaka Prefecture, 1-1 Gakuen-cho, Sakai, Osaka 593, ${ }^{2}$ Department of Veterinary Surgery, Faculty of Agriculture, The University of Tokyo, 1-1-1 Yayoi, Bunkyo-ku, Tokyo 113, and ${ }^{3)}$ Veterinary Medical Hospital, Faculty of Agriculture, Yamaguchi University, Yamaguchi 753, Japan

(Received 10 January 1994/Accepted 12 April 1994)

ABSTRACT. Computed tomography (CT) was performed on renal tumors (Wilms' tumor and renal cell carcinoma) and renal cysts in dogs and cats. CT images in renal tumors were well correlated with macroscopic findings, and contrast CT images were quite useful in differentiating tumoral regions from non-tumoral ones. On renal cysts, intravenous pyelography and ultrasonography were as effective as CT images in morphological diagnosis, but CT was considered to be superior for evaluating three-dimensional (3-D) relationships in complicated lesions.--KEY wORDs: computed tomography, renal cyst, renal tumor.

J. Vet. Med. Sci. 56(4): 813-816, 1994

Recent advances in imaging technology make computed tomography (CT) available in small animal medicine [4]. Renal CT images are thought to show the internal structure more clearly than radiography and ultrasonography, neither of which can clearly visualize the kidneys of an obese patient or the right kidney with costal interference $[5,8,10]$. The CT number objectively indicates the absorbance of $\mathrm{X}$-rays at a given renal parenchyma to identify the lesion $[2,5,10-12]$. In the present study, CT scanning in dogs and cats with kidney lesions was carried out to evaluate its diagnostic efficiency in renal diseases in small animals.

CT was performed on four patients with renal disease (one dog with a tumor, one dog with both a tumor and cysts, and two cats with cysts). Each dog and cat was premedicated with atropine sulfate $(0.025 \mathrm{mg} / \mathrm{kg})$ and xylazine $(2.0 \mathrm{mg} / \mathrm{kg})$ and anesthetized by an injection of sodium pentobarbital $(6-8 \mathrm{mg} / \mathrm{kg})$ before being placed in the supine position. The CT scanner used was a wholebody device type (CT-W 400-20, Hitachi Medico, Tokyo). Five- or 10-mm thickness, $120 \mathrm{kv}$ (fixed), 100-200 mA and 4.5 seconds exposure were used as scanning conditions. For contrast CT scanning, a bolus injection of a contrast medium (80\% w/v Angio-Conray, Daiichi Pharm. Co., Tokyo) at a dose of $1 \mathrm{~m} l / \mathrm{kg}$ of body weight was used immediately before scanning.

Figure 1 shows the CT images of the kidney in a female Japanese Spaniel dog (10 months old, $5 \mathrm{~kg}$ body weight) with nephroblastoma a few hours after intravenous pyelography. The parenchyma of the tumor was homogeneous with calcified (arrow) and radiolucent areas (arrowheads) (Fig. 1-a). The pelvis of the opposite kidney was enhanced by the contrast medium used in pyelography. The CT number of the tumoral parenchyma was slightly higher $(+52 \mathrm{HU})$ than that of the opposite normal kidney $(+37 \mathrm{HU})$. On contrast CT images (Fig. 1-b), the enhancement in the dorsal parenchyma was obvious, and was confirmed to be the renal pelvis histologically after surgical removal (arrowheads), but the tumoral parenchyma was not strongly enhanced. In the opposite normal kidney, the contrast medium strongly increased the CT number for the pelvis.
Although intravenous pyelography revealed the deformity and extent of the pelvis and ultrasonography revealed the solid mass lesions within the kidney (Fig. 2), which suggested the existence of a renal mass, the internal structure of the kidney was much clearer in CT images. In addition, the CT images of the renal tumor revealed the internal calcified and necrotic areas, which were confirmed macroscopically after surgical removal. It was reported that the CT number in Wilms' tumor was lower than that of the opposite kidney in humans [5], whereas the value was greater in this patient. The precise cause of this discrepancy was unclear.

Figure 3 shows the $\mathrm{CT}$ images of a female German Shepherd dog ( 8 years old, $30 \mathrm{~kg}$ body weight). From the CT images of the kidneys, bilateral cysts along with renal carcinoma were diagnosed. The tumoral mass was divided into areas of lower and higher density (Fig. 3-a), which were histologically confirmed as necrotic and tumoral tissues, respectively. The contrast CT images of the same slice revealed that the tumoral area was slightly enhanced whereas the necrotic tissues were not (Fig. 3-b). In addition, normal kidney tissue including the pelvis was more strongly enhanced than the non-necrotic tumoral tissues (arrowhead). The absence of a tumoral plug in the extended vessels was also confirmed on the CT image (arrow). Figure 4 shows the plain and contrast CT images of the caudal part of the same kidney. Renal cysts observed to be round in shape and of lower density were minimally enhanced in contrast CT. A significant difference between the CT numbers for the tumoral necrotic tissues and renal cysts was observed in this case, but they could be differentiated by their shapes on CT images. Although intravenous pyelography revealed the pelvis, and ultrasonography revealed the internal structure of the tumor with the mixture of high and low echo dense areas and some capsulated water-dense areas (Fig. 5) in this case, CT was superior in clearly revealing the margination, the internal structure, and their relationships in three dimensions.

Figures 6 and 7 show the CT images and an ultrasonograph of two Persian cats (9-year-old female in Fig. 6 and 10-year-old spayed male in Fig. 7), with perirenal cysts. 


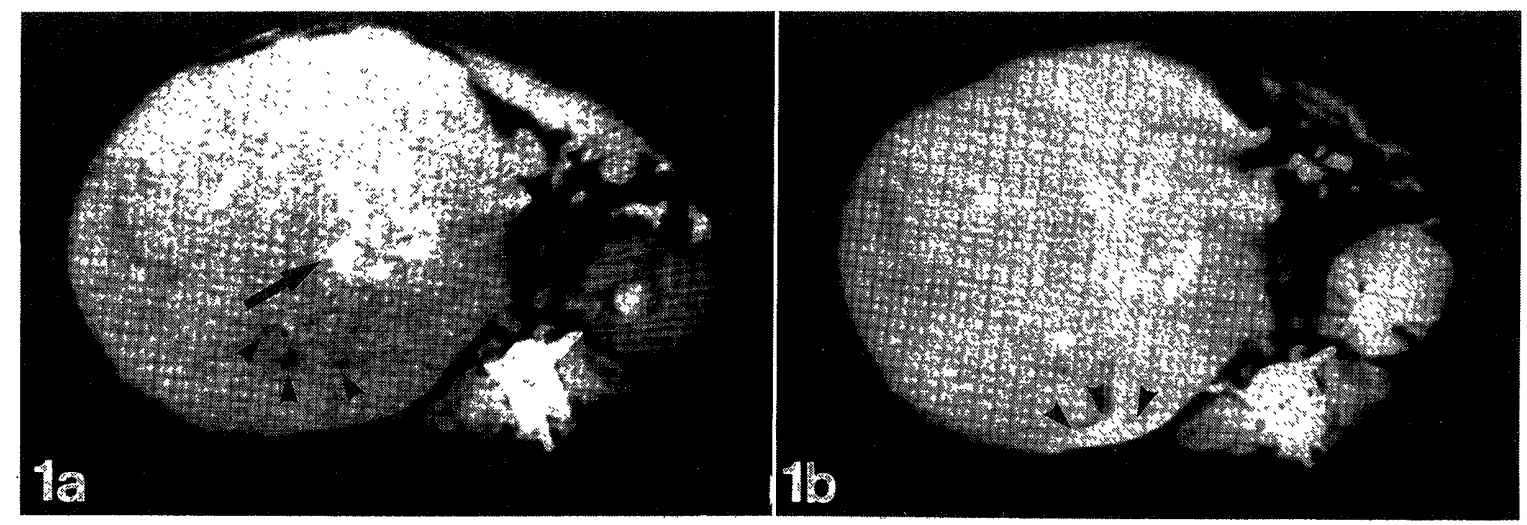

Fig. 1. CT images of a dog with nephroblastoma. a) Calcification (arrow) and radiolucent portions (arrowheads) are seen. b) The renal pelvis (arrowheads) is enhanced in a contrast CT image.

Perirenal and renal cysts on plain CT images were identified as of low-density, thin-walled area without internal structures, and no enhancement was seen in the contrast study (Fig. 6-a, Fig. 7). The wall thickness of the perirenal cyst, which was microscopically identified as fibrous hypertrophy of the serosa, was quite thin on CT images. If the cyst contained coagulated fibrin, pus or

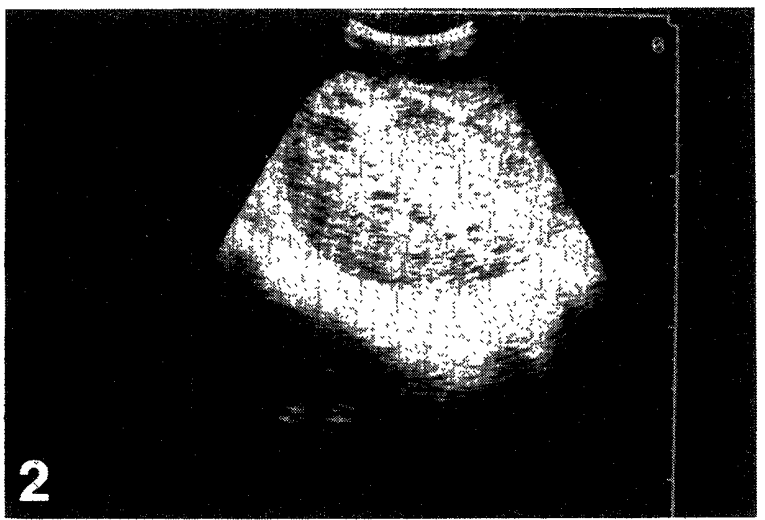

Fig. 2. An ultrasonograph of the right kidney showing the renal mass in the same patient as in Fig. 1.
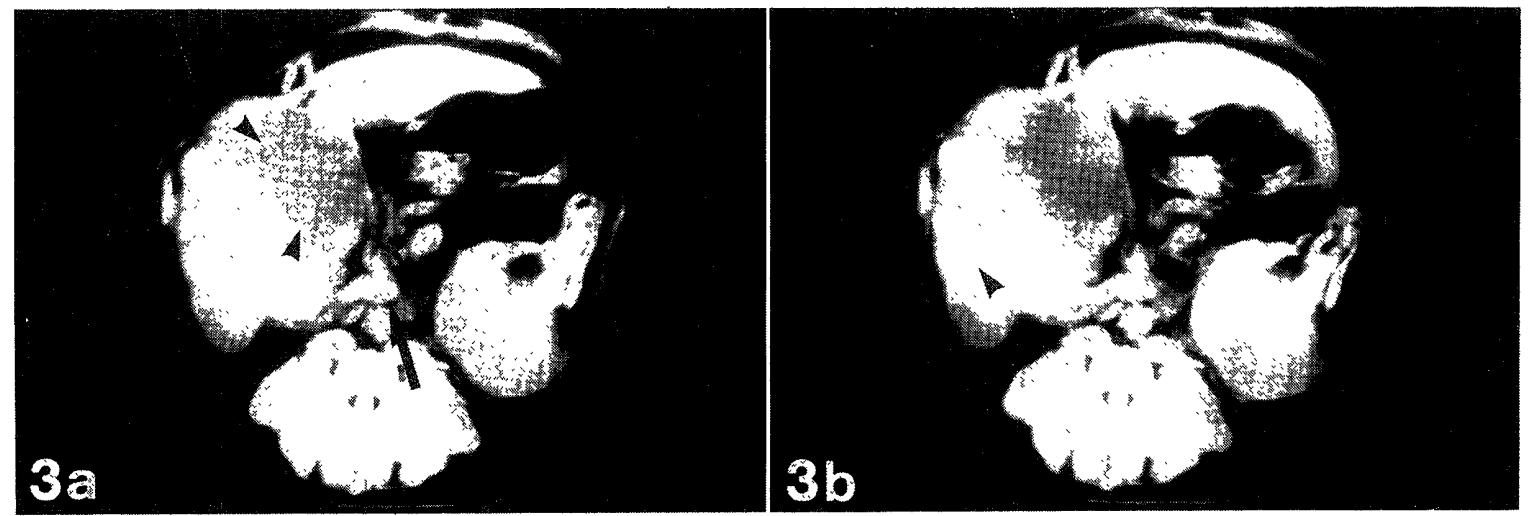

Fig. 3. CT images of renal cell carcinoma in a German Shepherd dog. a) Plain CT scan at the middle of the right kidney revealed necrosis (arrowheads) and unaffected extended renal vein (arrow). b) A contrast CT image of the same slice revealed the slightly enhanced (tumoral mass) and hardly enhanced (necrotic tissue) areas. In addition, normal kidney tissue and the pelvis was strongly enhanced (arrowhead). some other substances, the internal CT values rose, unlike the levels which are generally similar to those for water [10]. Moreover, the characteristic features differentiating a cyst from tumor necrosis are the thin-walled structure of the cyst and the sharp margination [10]. In the cases shown in Figs. 6 and 7 , intravenous pyelography and ultrasonography (Fig. 6-b) clearly revealed the cysts and the position of each. They were comparable to CT images in determining the number of renal cysts and the relationship of perirenal cysts to the kidney.

The density of the renal tumor is generally different from the density of opposite normal kidney, and highdensity structures (hemorrhage and calcification) or lowdensity ones (necrosis and fat) are occasionally found [5]. When a lesion is not distinguishable, an enhanced CT scan should be carried out to contrast it with the normal parenchyma, because the enhanced effect is revealed in the normal parenchyma which maintains its functioning nephrons $[3,5]$.

In terms of CT images of renal and perirenal cysts, the present study was compatible with diagnostic features such as the absence of internal structure and no enhancement effect $[1,2,7,9]$. On the other hand, intravenous pyelography can provide useful information about renal 

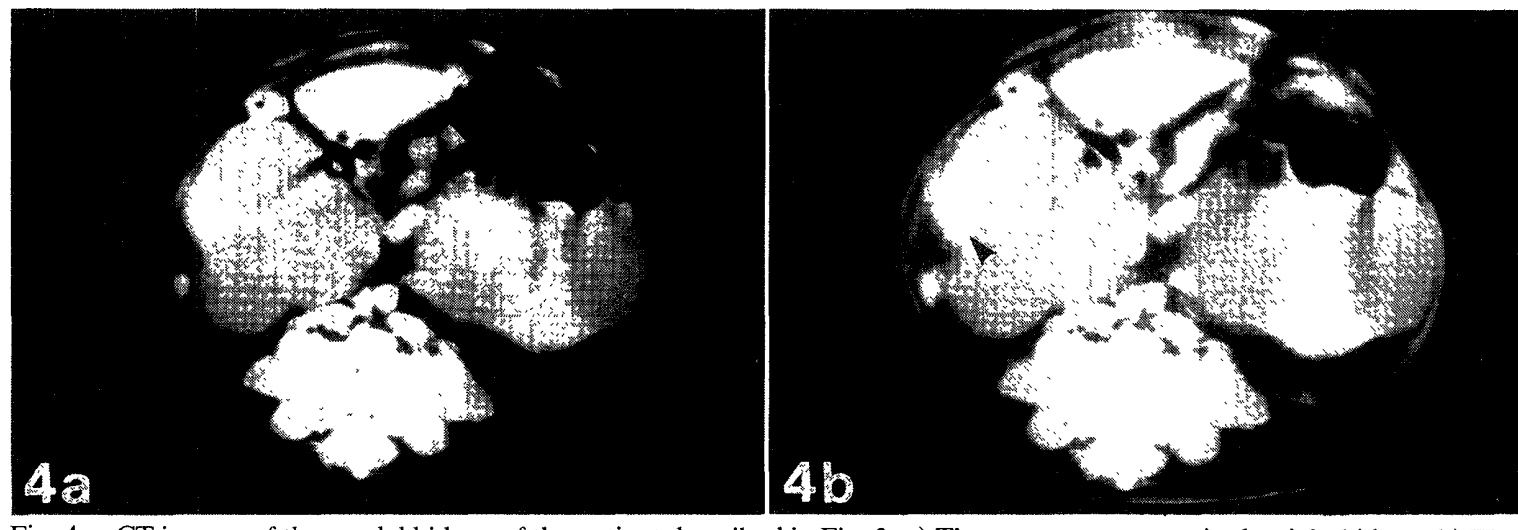

Fig. 4. CT images of the caudal kidney of the patient described in Fig. 3. a) Three cysts were seen in the right kidney. b) The renal pelvis (arrowhead) was enhanced in a contrast CT image.

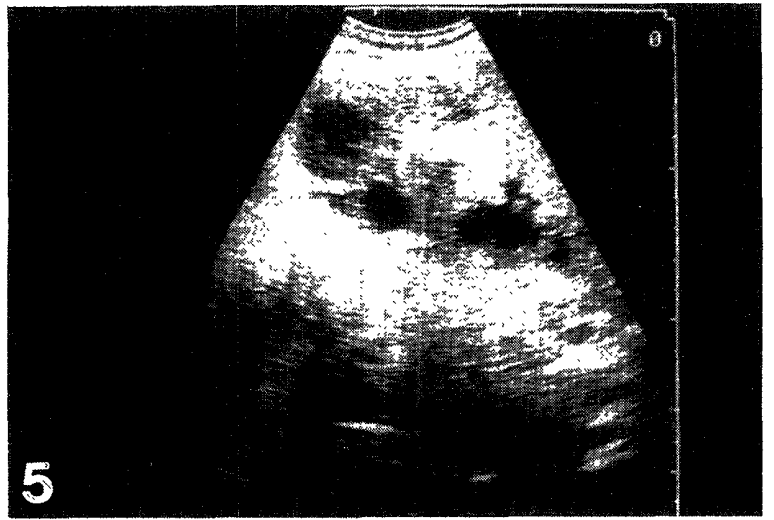

Fig. 5. An ultrasonograph of the right kidney in the patient in Figs. 3 and 4.

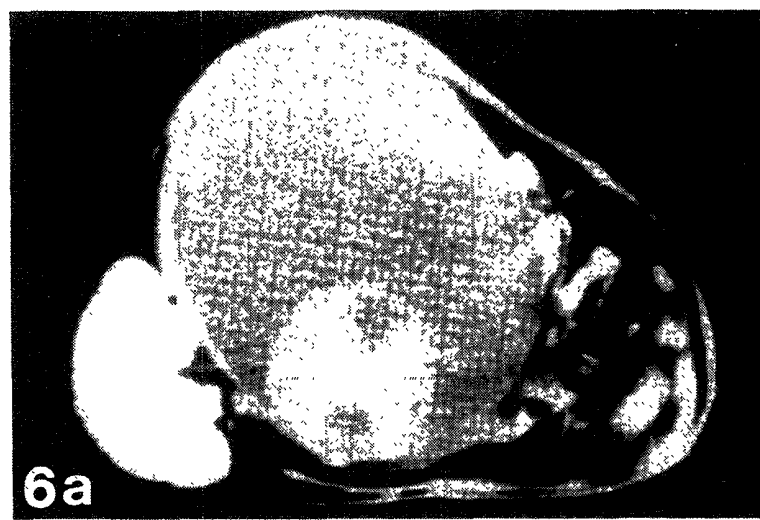

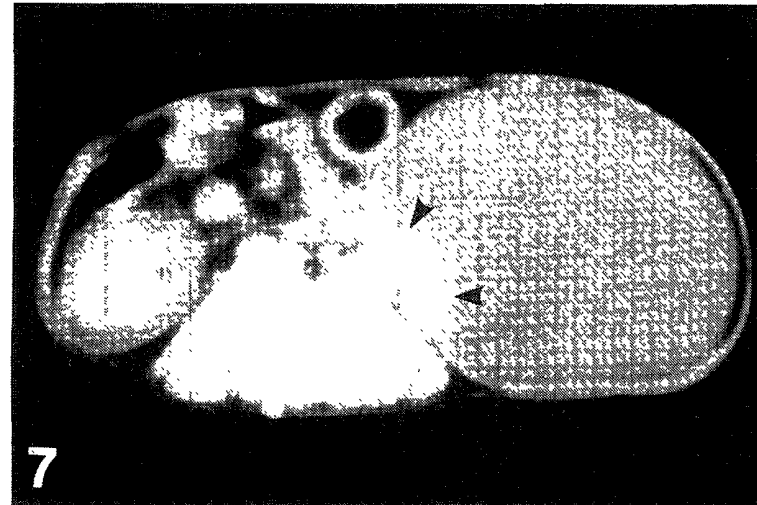

Fig. 7. A contrast CT image of a perirenal cyst in a Persian cat. Renal parenchyma (arrowheads) was strongly enhanced.

Fig. 6. A contrast CT image and an ultrasonograph of renal and perirenal cyst in a Persian cat. a) A large cyst with a thin wall surrounding a normal kidney is seen. b) A similar finding was obtained in a ultrasonograph.

function as well as renal structure [3], and the section of kidney scanned by ultrasonography correlates with its macroscopic findings [6]. However, CT has been reported to be more accurate than ultrasonography $[8,10]$. In the present study, intravenous pyelography and ultrasonography provided good images for diagnosing renal cyst compared with tumor whose images were inferior for determining the relationship of the tumor to the kidney, and the degree of infiltration of the tumor into the perirenal tissue.

In conclusion, CT examination is useful for renal tumors and cysts in small animal practice, as a means to clearly visualize the internal structure. It proved to be especially useful in distinguishing the tumoral infiltration from the non-tumoral parts of the kidney, and to understand the positional relationships in three dimen- 
sions of lesional complications.

\section{REFERENCES}

1. Brennan, R. E., Curtis, J. A., Pollack, H. M., and Weinberg, I. 1979. Invest. Radiol. 14: 239-245.

2. Burgener, F. A. and Hamlin, D. J. 1981. Am. J. Roentgen. 137: $351-358$.

3. Feeney, D. A., Barber, D. L., and Osborne, C. A. 1982. Vet. Radiol. 23: 42-45.

4. Feeney, D. A. and Hardy, R. M. 1992. pp. 10-18. In: Current Veterinary Therapy XI. W. B. Saunders, Philadelphia.

5. Fishman, E. K., Hartman, D. S., Goldman, S. M., and Siegelman, S. S. 1983. J. Comput. Assist. Tomogr. 7: 659-665.
6. Konde, L. J., Wrigley, R. H., Park, R. D., and Lebel, J. L. 1984. Vet. Radiol. 25: 173-178.

7. Madewell, J. E., Goldman, S. M., Davis Jr., C. J., Hartman, D. S., Feigin, D. S., and Lichtenstein, J. E. 1983. Radiology 146: 309-321.

8. Magilner, A. D. and Ostrum, B. J. 1978. Radiology 126: 715-718.

9. Parikh, P. U., Korobkin, M., McClennan, B. L., Platt, J. F., and Weingarten, B. J. 1992. J. Comput. Assist. Tomogr. 16: $586-588$.

10. Sagel, S. S., Stanley, R. J., Levitt, R. G., and Geisse, G. 1977. Radiology. 124: 359-370.

11. Shirkhoda, A. and Lewis, E. 1987. Radiology. 162: 353-357.

12. Tammela, T. L. J., Leinonen, A. S. S., and Kontturi, M. J. 1991. Scand. J. Nephrol. 25: 283-286. 